\title{
The ATLAS Run 2 Trigger: Design, Menu, Performance and Operational Aspects
}

\author{
Joana Machado Miguéns*, on behalf of the ATLAS Collaboration \\ University of Pennsylvania \\ E-mail: jmiguens@cern.ch
}

The LHC, at design capacity, has a bunch-crossing rate of $40 \mathrm{MHz}$ whereas the ATLAS experiment is limited by offline computing and storage to an average recording rate of physics events of $\sim 1 \mathrm{kHz}$. To reduce the rate of events but still maintain high efficiency of selecting rare events such as possible physics signals beyond the Standard Model, a two-level trigger system is used in ATLAS. Events are selected based on physics signatures such as presence of energetic leptons, photons, jets or large missing energy. Despite the limited time available for processing collision events, the trigger system is able to exploit topological information, as well as using multi-variate methods. In total, the ATLAS trigger system consists of about two thousand different individual trigger selection strategies. The ATLAS trigger menu specifies which triggers are used during data taking and how much rate a given trigger is allocated. This menu reflects not only the physics goals of the collaboration but also takes into consideration the instantaneous luminosity of the LHC and the design limits of the ATLAS detector, the data acquisition system and the offline processing Tier-0 farm. We describe the criteria for designing the ATLAS trigger menu used for the LHC Run 2 period. Furthermore, we discuss how the trigger menu is deployed online, through different phases: validation before being used online, decision on prescale values for different triggers (ahead of running, or online in case of sudden rate changes due to changes in data-taking conditions), and monitoring during data taking itself. The performance of the high-level trigger algorithms used to identify leptons, hadrons and global event quantities is presented. These objects are crucial for event selection relevant to a wide range of physics analyses, of which a few examples are given.

38th International Conference on High Energy Physics

3-10 August 2016

Chicago, USA

${ }^{*}$ Speaker. 


\section{Introduction}

The trigger system is an essential component of any hadron collider experiment as it is responsible for deciding whether or not to keep an event from a given bunch-crossing for later study. During Run 1 of the Large Hadron Collider (LHC), the trigger system [1] of the ATLAS experiment [2] was operated efficiently at instantaneous luminosities of up to $8 \times 10^{33} \mathrm{~cm}^{-2} \mathrm{~s}^{-1}$ and primarily at centre-of-mass energies, $\sqrt{s}$, of $7 \mathrm{TeV}$ and $8 \mathrm{TeV}$. Run 2 of the LHC presents a challenging environment with the increase in $\sqrt{s}$ to $13 \mathrm{TeV}$ and instantaneous luminosities surpassing the design maximum of $10^{34} \mathrm{~cm}^{-2} \mathrm{~s}^{-1}$. This instantaneous luminosity also corresponds to a large number of proton-proton ( $p p$ ) collisions per bunch-crossing (pile-up). Substantial modifications and upgrades of the trigger system were done after Run 1 and sophisticated trigger strategies are now being employed to ensure stable and reliable running and that the physics goals of ATLAS are met in Run 2 [3].

This document summarizes the design of the ATLAS trigger, presents the trigger menu strategy, discusses operational aspects and shows examples of trigger performance assessment in Run 2.

\section{Run 2 trigger system}

The Trigger and Data Acquisition (TDAQ) system of ATLAS consists of a hardware-based first level (L1) and a software-based high level trigger (HLT). The L1 trigger is implemented in fast custom-made electronics, runs with a fixed latency of $2.5 \mu \mathrm{s}$ and reduces the event rate from $40 \mathrm{MHz}$ to $100 \mathrm{kHz}$. The L1 trigger decision is formed by the Central Trigger Processor (CTP), which receives information from the L1 calorimeter (L1Calo) and L1 muon (L1Muon) triggers. All three components were upgraded for Run 2 and a new topological trigger (L1Topo), programmed to perform selections based on geometric or kinematic association between trigger objects received from L1Calo or L1Muon, has been recently included and is currently being commissioned.

At the HLT offline-like reconstruction algorithms run in a single farm of $\sim 40000$ processors and a decision is formed typically within $300 \mathrm{~ms}$. Hardware-based tracking capabilities will be provided by the Fast Tracker (FTK) currently in test-phase. Events accepted by the HLT are written into different data streams. Those meant to be used for physics analysis, for which all the subdetector data is stored, are sent to a single Main stream at an average rate of $1 \mathrm{kHz}$ limited by the offline computing model. Partial event building (EB) streams have smaller event sizes, as only relevant sub-detector data is stored, and thus can run at higher rates. Events in these streams are used for detector calibration, monitoring and Trigger Level Analysis. Figure 1 shows the HLT stream rates during a typical LHC fill and the average bandwidth consumption of these of these streams as a fraction of the total bandwidth.

\section{Trigger menu}

The trigger menu defines the list of L1 and HLT triggers used for data-taking and includes: primary triggers used for physics measurements and typically running unprescaled; support triggers used for efficiency and performance measurements and running prescaled at lower rates; alternative triggers running other selections and largely overlapping with primary triggers; backup triggers with tighter selections and lower expected rates for use in case of an increase in instantaneous luminosity or other changes in data-taking conditions; and calibration triggers, running at high rate but using partial event building. 

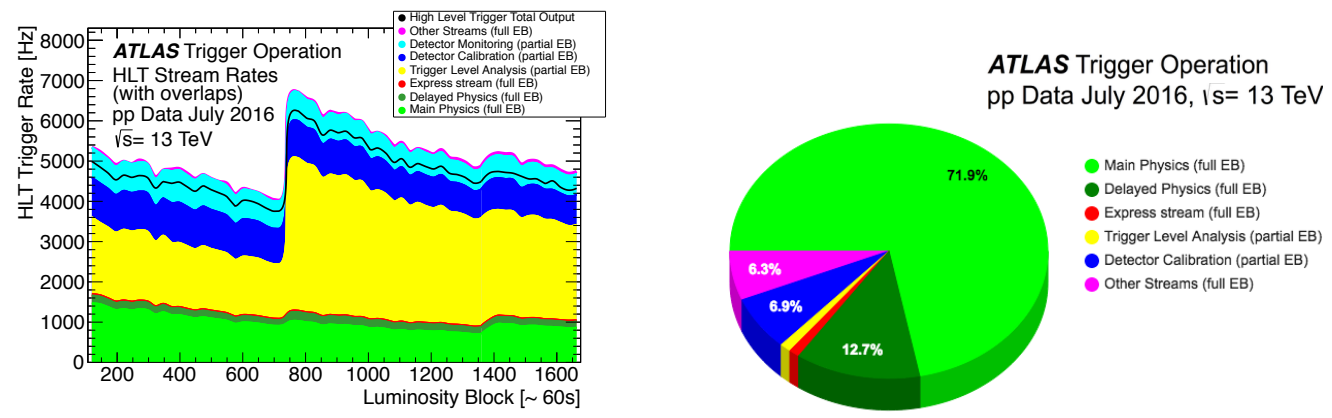

Figure 1: (Left) HLT stream rates as a function of the number of luminosity blocks (which last $60 \mathrm{~s}$ on average), in a fill taken in July 2016 with a peak luminosity of $1.02 \times 10^{34} \mathrm{~cm}^{-2} \mathrm{~s}^{-1}$ and an average (peak) pile up of 24.2 (35). Stream overlaps are only accounted for in the HLT total output rate displayed by the black line. (Right) Contribution of the HLT streams the total output bandwidth for the same fill [4].

The current trigger menu consists of over 2000 trigger selection strategies. The composition of the menu is driven by the physics goals of ATLAS and constrained by the rate and bandwidth limitations of the detector, TDAQ system, and offline computing. Primary triggers cover a variety of signatures, including electrons, photons, muons, tau leptons, $(b$-)jets and missing transverse energy, and are used in several Standard Model measurements (e.g. top and Higgs) as well as searches for new physics. Specialized triggers are generally allocated a rate of $O(1 \mathrm{~Hz})$, whereas more generic triggers aim at $O(10 \mathrm{~Hz})$. Single electron and single muon triggers run at $O(100 \mathrm{~Hz})$ as they serve many analyses. Approximately $20 \%$ of HLT bandwidth is dedicated to supporting triggers.

A handful of trigger menus have been designed with different compositions and trigger thresholds assuming different peak luminosities. Primary triggers are kept stable in each menu, which is desirable for physics analyses. This strategy allows the experiment to adjust to the changing conditions during the LHC instantaneous luminosity ramp-up, maximizing the physics output and fitting within the constraints of the system.

The trigger menu design is complex, therefore tools have been developed to validate the trigger algorithms which will ensure the menu fits within data-taking limitations. A special dataset is collected upon changes in the data-taking conditions. These events can be weighted into an effective zero-bias dataset, i.e. events minimally biased by the trigger pre-selection, that can be used for trigger rate predictions [5]. Furthermore any development is carefully validated by running the full trigger menu offline in this enhanced-bias dataset, before being deployed online for data-taking in the selective mode.

\section{Operational aspects}

The trigger menu is typically deployed online for data-taking with different sets of prescales. The L1 and HLT trigger rates for different signatures during a typical LHC fill can be seen in Figure 2. Single electron and single muon triggers contribute a large fraction to the total rate. As the luminosity decreases with time the resource usage is optimized by increasing the rate of supporting triggers or enabling additional triggers later in the fill.

Given the complexity of the trigger menu and the system overall, careful monitoring of the trigger rates online is essential to ensure high efficiency data-taking. Figure 3 shows examples of 

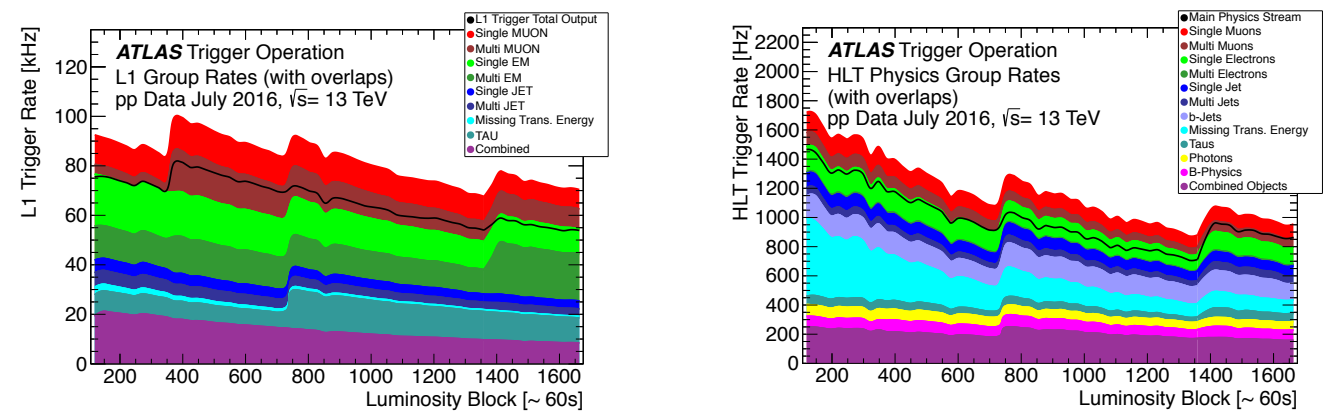

Figure 2: L1 (left) and HLT (right) physics trigger rates grouped by trigger signatures as a function of the number of luminosity blocks in a fill taken in July 2016 with a peak luminosity of $1.02 \times 10^{34} \mathrm{~cm}^{-2} \mathrm{~s}^{-1}$ and an average (peak) pile up of 24.2 (35). Due to overlaps the sum of the individual groups is higher that the total L1 rate (left) and Main physics stream rate (right), which are shown as black lines. The rates periodically increase due to change of prescales to optimise the resource usage [4].

the online rates of some primary L1 triggers monitored during a typical LHC fill. The online rates are displayed with predictions obtained by scaling the rates measured in previous fills according to the actual luminosity. This comparison offers a powerful tool to recognize in real time potential anomalies with the trigger during data-taking. Further monitoring of the performance of the trigger during data-taking is achieved by regularly applying automatic data quality (DQ) checks, based on standard histogram analysis techniques, to over 500 distributions of quantities reconstructed by the HLT algorithms.
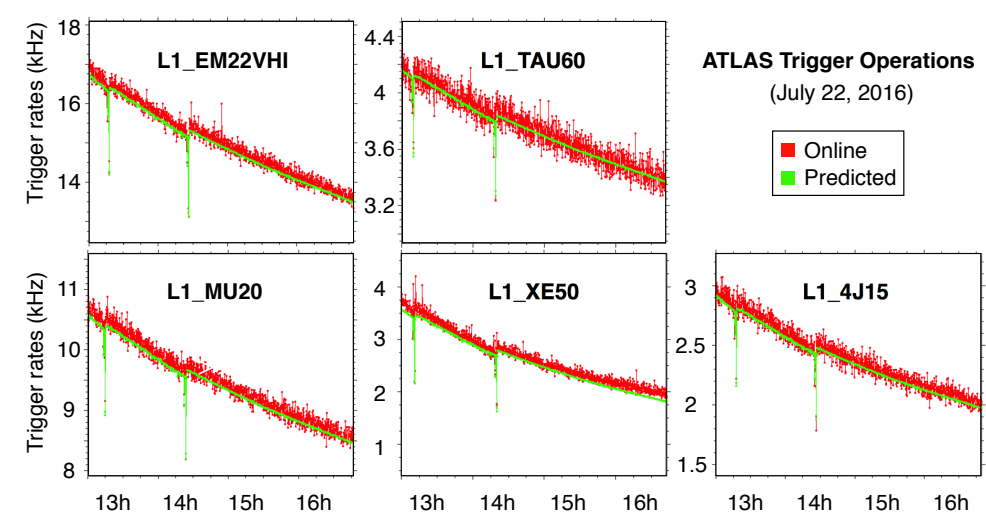

Figure 3: Level-1 trigger rates online (red) compared with predictions based on luminosity-scaling (green) for five algorithms noted in the plot. The downward spikes correspond to the luminosity optimization done by the LHC [4].

\section{Performance}

Several improvements have been made in the muon trigger reconstruction for Run 2. In 2016 the acceptance of the L1 muon trigger detectors in the barrel region was increased by $4 \%$ with newly installed chambers in the so-called feet region of the muon system. The impact can be seen in Figure 4 (left), displaying the efficiency of a L1 muon trigger in the barrel region with respect to the offline muon selection as a function of $\phi$, for data collected in 2015 and in 2016. The improvement in the efficiency is visible for $\phi \sim-2$. Figure 4 (right) shows the absolute efficiencies of a L1 muon trigger and an HLT muon trigger with an isolation requirement, as well as the HLT 
efficiency with respect to $\mathrm{L} 1$, as a function of the transverse momentum of the offline muon in the endcap region. The efficiency at L1 is close to $90 \%$ due to the geometrical acceptance. At the HLT the efficiency relative to $\mathrm{L} 1$ is close to $100 \%$.
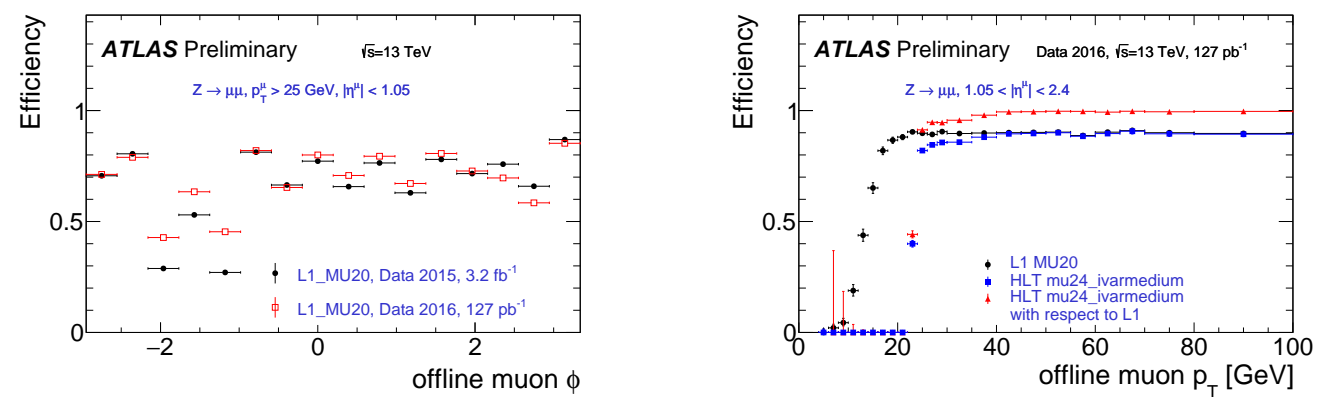

Figure 4: (Left) Absolute efficiency of the L1_MU2 0 trigger in 2015 (black) and in 2016 (red) plotted as a function of $\phi$ of offline muon candidates in the barrel detector region. (Right) Absolute efficiency of the L1_MU2 0 trigger and the absolute and relative efficiencies of the mu24_ivarmedium HLT trigger, as a function of the transverse momentum of the offline muon in the endcap region. All efficiencies are measured in data collected in 2016 using a tag-and-probe method with $Z \rightarrow \mu \mu$ candidates [6].

For Run 2 the speed of calorimeter clustering algorithms was improved by a factor of 2 and the timing of the calorimeter data unpacking was improved by a factor of 7 . As a result jet trigger algorithms at the HLT are able to perform a full scan of the detector. Figure 5 (left) shows the efficiency of several HLT jet triggers, as a function of the offline jet transverse momentum. The sharp turn-on curves seen in a very large $p_{\mathrm{T}}$ range are due to the good agreement between the energy scale of HLT and offline jets. The increased pile-up in 2016 has a small impact in the efficiency of these jet triggers.

The missing transverse energy $\left(E_{\mathrm{T}}^{\mathrm{miss}}\right)$ trigger rates are severely affected by detector noise and mismeasurements, and generally increase faster than linearly with pile-up. At the HLT several $E_{\mathrm{T}}^{\text {miss }}$ reconstruction algorithms have been implemented using only energy measurements in the calorimeter. The efficiencies for different $E_{\mathrm{T}}^{\text {miss }}$ triggers at the HLT, as well as the efficiency for the L1_XE50 trigger at L1, are shown in Figure 5 (right) as a function of the offline $E_{\mathrm{T}}^{\text {miss }}$. The "mht" algorithm, which reconstructs $E_{\mathrm{T}}^{\text {miss }}$ by summing the $p_{\mathrm{T}}$ of the jets reconstructed at the HLT, is currently used by default as it shows better performance.
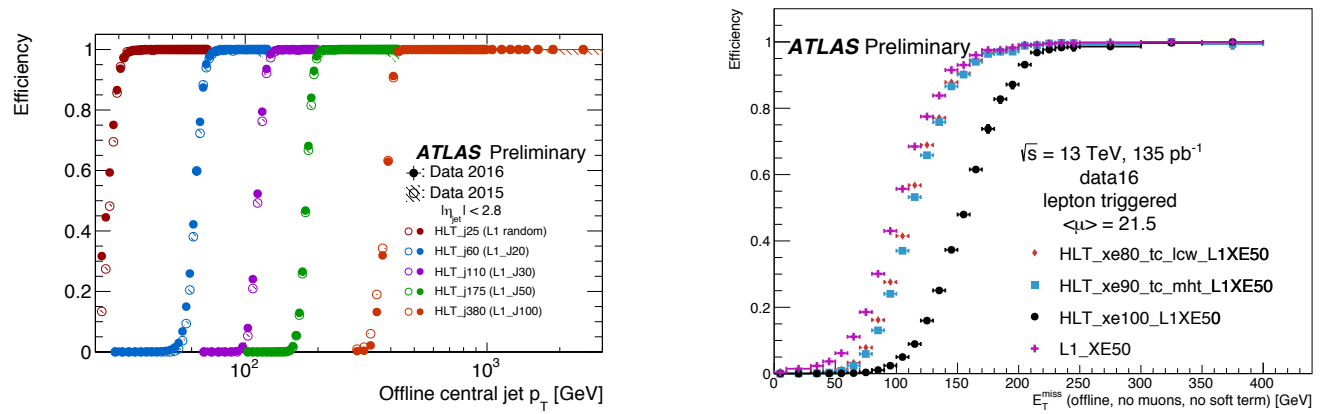

Figure 5: (Left) Efficiencies for HLT single-jet triggers as a function of leading offline jet $p_{\mathrm{T}}$ for jets with $|\eta|<2.8$. Triggers in 2016 (filled circles) become fully efficient at the same point as was observed in 2015 (open circles), despite higher levels of pileup. (Right) Efficiency as a function of offline $E_{\mathrm{T}}^{\text {miss }}$ for three different $E_{\mathrm{T}}^{\text {miss }}$ trigger algorithms, using early $p p$ collision data from 2016. All three algorithms are seeded by a $\mathrm{L} 1$ trigger algorithm with a nominal threshold of $50 \mathrm{GeV}$ which is also shown $[7,8]$. 
One of the major improvements in Run 2 for the HLT electron and photon reconstruction is in the calibration of the energy of the electromagnetic clusters, which uses a multivariate technique to correct the detector response. Furthermore, electron identification at the HLT also relies on a multivariate technique based on a likelihood (LH) discriminant that provides an increased signal purity. The efficiency curves for the primary single-electron trigger at L1 and at the HLT are shown in Figure 6 (left) as a function of the transver energy $\left(E_{\mathrm{T}}\right)$ of the offline reconstructed electrons. The HLT $E_{\mathrm{T}}$ threshold of $24 \mathrm{GeV}$ was maintained thanks to the use of a tight identification working point and the use of isolation criteria. High HLT trigger efficiency as a function of $E_{\mathrm{T}}$ of the offline reconstructed photon is shown in Figure 6 (right) for inclusive high $E_{\mathrm{T}}$ photon triggers, complemented by lower $E_{\mathrm{T}}$ diphoton trigger selections.
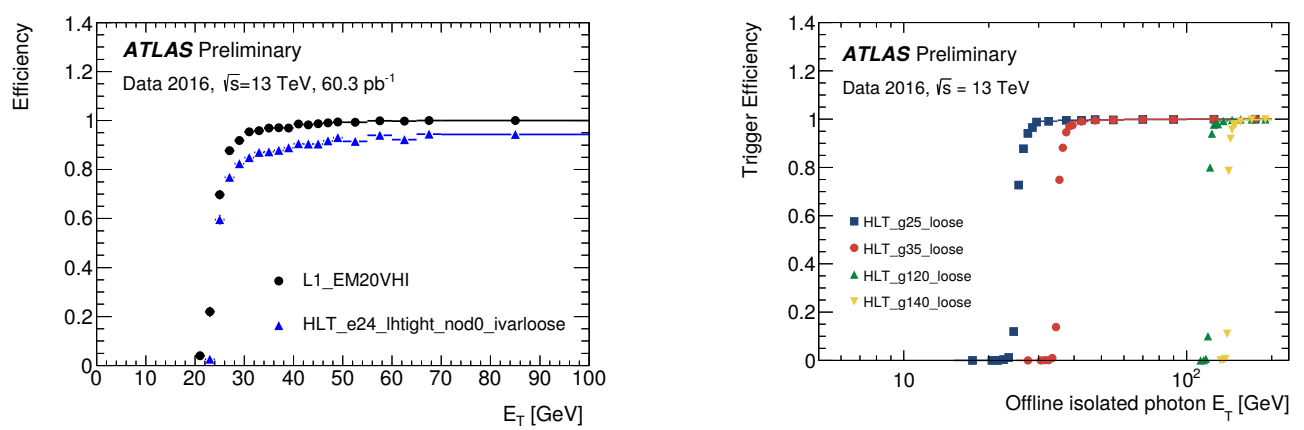

Figure 6: (Left) Efficiency of the L1_EM2OVHI and the e24_lhtight_nod0_ivarloose electron triggers as a function of the $E_{\mathrm{T}}$ of the offline reconstructed electrons, measured in data with a tag-and-probe method using $Z \rightarrow e e$ decay. (Right) Efficiency of photon triggers requiring transverse energies greater than $25,35,120$ and $140 \mathrm{GeV}$ and loose photon identification criteria as a function of the offline photon $E_{\mathrm{T}}$, measured using events recorded with a L1 trigger requiring an electromagnetic cluster with $E_{\mathrm{T}}>15 \mathrm{GeV}$ [9].

\section{Conclusions}

The LHC Run 2 conditions pose a challenge to the ATLAS trigger system, which went through fundamental hardware and software improvements after Run 1. A well designed trigger menu ensures the physics goals of the ATLAS experiment are met, while respecting the limitations of the system. The ATLAS trigger system was successfully commissioned in 2015 and has been operating smoothly in 2016 despite the challenging running conditions. New studies using data collected this year show the overall great performance of the trigger. Further improvements are planned for next year with the commissioning of FTK and the use of L1Topo triggers. In conclusion, the ATLAS trigger system is ready to exploit the full potential of the LHC.

\section{References}

[1] ATLAS Collaboration, Eur. Phys. J. C72 (2012) 1849 [hep-ex/1110.1530].

[2] ATLAS Collaboration, JINST 3 (2018) S08003.

[3] ATLAS Collaboration, ATL-DAQ-PUB-2016-001 [cds.cern. ch/record/2136007].

[4] [http://twiki.cern.ch/twiki/bin/view/AtlasPublic/TriggeroperationPublicResults].

[5] ATLAS Collaboration, ATL-DAQ-PUB-2016-001 [cds . cern. ch/record/2223498].

[6] [http://twiki.cern.ch/twiki/bin/view/AtlasPublic/MuonTriggerPublicResults].

[7] [http://twiki.cern.ch/twiki/bin/view/AtlasPublic/JetTriggerPublicResults].

[8] [http://twiki.cern.ch/twiki/bin/view/AtlasPublic/MissingEtTriggerPublicResults].

[9] [http://twiki.cern.ch/twiki/bin/view/AtlasPublic/EgammaTriggerPublicResults]. 